\title{
Debatte über Renten: Replik und Erwiderung
}

\author{
In der Juniausgabe 2021 veröffentlichte der Wirtschaftsdienst einen Aufsatz mit dem Titel \\ „Beitragsfinanzierung im ,demografiegestressten“ Rentensystem möglich“ von Ernst Niemeier. \\ Martin Werding vertritt in einer Replik eine andere Auffassung, im Anschluss erläutert Ernst \\ Niemeier seinen Standpunkt in einer Erwiderung.
}

\author{
Martin Werding
}

\section{Warum das Rentenniveau nicht fixiert werden sollte: ein Kommentar}

Im Wirtschaftsdienst 6/2021 weist Ernst Niemeier (2021) Überlegungen der Kommission „Verlässlicher Generationenvertrag“ (2020) und des Wissenschaftlichen Beirats beim BMF (2020) zurück, nach denen das Niveau der gesetzlichen Renten in Zukunft sinken sollte, um ständig steigende Beitragssätze zu vermeiden. Sein zentrales Argument ist, dass ein konstantes Rentenniveau auf Dauer „tragbar" sei, weil steigende Beiträge den erwarteten Anstieg der Reallöhne nicht vollständig abschöpfen. Diese sehr einfache Sicht fordert einen kritischen Kommentar heraus.

Ökonomen beurteilen meist zurückhaltend, ob Zukunftsszenarien der hier betrachteten Art realisierbar sind oder nicht. Eher rechnen sie Konsequenzen durch, die z.B. ein konstantes Sicherungsniveau der gesetzlichen Rentenversicherung (GRV) hätte, und weisen auf absehbare Nebenwirkungen hin. So komme ich in einer von Niemeier (2021) gleichfalls zitierten Studie zu dem Schluss, dass sich eine Fixierung des Rentenniveaus in der mittlerweile unmittelbar bevorstehenden, akuten Phase des demografischen Wandels spätestens ab 2030 „kaum durchhalten“ lässt (Werding, 2018, 35). Zuvor wird in der Studie abgeschätzt, dass der GRV-Beitragssatz bei einem Sicherungsniveau von mindestens $48 \%$ (in Klammern: $50 \%$ ) bis 2043 (2038) die $25 \%$-Grenze überschreiten und bis 2080 weiter auf 32,5\% (33,6\%) steigen müsste (Werding, 2018, 29 f.).

Die Nettolöhne sozialversicherungspflichtig Beschäftigter würden unter den dabei getroffenen Annahmen - wie von Niemeier (2021, 456 f.) betont - in der Tat kontinuierlich steigen, wenn auch deutlich langsamer als die Bruttolöhne. Fragen kann man daher nach den Maßstäben dafür,

(c) Der/die Autor:in(nen) 2021. Open Access: Dieser Artikel wird unter der Creative Commons Namensnennung 4.0 International Lizenz veröffentlicht (creativecommons.org/licenses/by/4.0/deed.de).

Open Access wird durch die ZBW - Leibniz-Informationszentrum Wirtschaft gefördert. dass viele Expert:innen, neuerdings auch die Mitglieder des Wissenschaftlichen Beirats beim BMWi (2021), eine Fixierung des Rentenniveaus für schwer realisierbar und keinesfalls empfehlenswert halten.

\section{Fehlende Maßstäbe}

Verlässliche Maßstäbe für die gegenteilige Schlussfolgerung bleibt auch Niemeier (2021) schuldig. Er sagt in seinem Beitrag nicht einmal, wie er das „Rentenniveau“ definiert, das er dauerhaft gern bei „z.B. mindestens $50 \%$ “ (Niemeier, 2021, 459) sähe. Die angegebenen Zahlen und Zitate legen nahe, dass er damit das (Standard-)Sicherungsniveau vor Steuern meint, auf das sich auch die derzeit geltende „Haltelinie“ bezieht.

Diese Größe sagt wenig über die tatsächliche Versorgung der Rentner:innen aus, unter anderem weil sie durch den Abzug der Sozialversicherungsbeiträge von Standardrente und Durchschnittsentgelt der aktiven Versicherten, aber ohne Berücksichtigung von Steuern, auf halbem Weg zwischen einem Brutto- und einem Netto-Rentenniveau stehen bleibt. Der Schritt in die Richtung einer Netto-Definition ist allerdings wichtig. Die Forderung nach einem konstanten Brutto-Rentenniveau, das in den von 1957 bis 1991 gültigen Rentenanpassungsformeln angelegt war, kann in der Tat die Grenzen des rechnerisch Möglichen verletzen. Bei extremen demografischen Verschiebungen macht ein konstantes Brutto-Rentenniveau nämlich Beitragssätze von über $100 \%$ erforderlich. Bei konstantem Netto-Rentenniveau konvergieren sie im Extremfall „nur“

Prof. Dr. Martin Werding ist Professor für Sozialpolitik und öffentliche Finanzen an der RuhrUniversität Bochum. 
gegen $100 \%$. Bei realistischen Annahmen bleiben sie erkennbar darunter, sodass ein genaueres Durchrechnen immerhin lohnt.

Verlässlich ist der so präzisierte Maßstab - „steigen die realen Nettoentgelte bei höherer Beitragsbelastung noch an?" - aber weiterhin nicht. Im Rentenrecht gibt es nämlich keinen Mechanismus, der dies gewährleistet. Wie sich das Sicherungsniveau auf den Beitragssatz der GRV auswirkt, ist bei gegebener demografischer Entwicklung weitestgehend unabhängig davon, wie stark die Bruttolöhne der aktiven Versicherten steigen (dazu etwa Werding, 2018, 24 f.). Wenn diese Löhne real oder sogar nominal unverändert blieben, müsste der GRV-Beitragssatz bei konstantem Sicherungsniveau ganz genauso ansteigen wie zuvor ausgeführt, und die Nettolöhne würden sinken.

\section{Maßstab I: intergenerationelle Lastverteilung}

Das Rentenniveau trotz der klar absehbaren demografischen Alterung zu fixieren, lässt den Beitragssatz der GRV stark steigen - mit großem Tempo bis etwa 2045, danach aber fast ungebrochen weiter. Erst nach 2060 flacht der Anstieg aus heutiger Sicht etwas ab. Wie hoch genau der Beitragssatz dabei wird, lässt sich wegen zahlreicher Unsicherheiten nicht sagen. Die zuvor genannten Zahlen geben aber eine Vorstellung von der Größenordnung solcher Effekte.

Wenn jüngere und zukünftige Versicherte für auf den ersten Blick gleichwertige Leistungen der GRV einen deutlich größeren Teil ihres laufenden Einkommens aufwenden müssen als heutige Rentner:innen und rentennahe Jahrgänge, erleiden sie Nachteile, die sich z.B. anhand der „internen Renditen“ des gesetzlichen Rentensystems (Sozialbeirat, 2004, Tz. 100-136; Sachverständigenrat 2016, Tz. 667-681) oder der darin enthaltenen „impliziten Steuer" (Fenge und Werding, 2004) messen lassen. Diese Nachteile treffen Alterskohorten, die für den demografischen Wandel definitiv nichts können. Ihre Möglichkeiten, eine Altersvorsorge aufzubauen, die weniger von der demografischen Entwicklung abhängt, werden dadurch substanziell eingeengt. Der Verweis auf zukünftige Produktivitäts- und Einkommenssteigerungen, die die Jüngeren irgendwie auch den Älteren verdanken (Niemeier, 2021, 457 f.), ist vage und unsicher. In Zeiten noch nicht voll absehbarer Folgen des Klimawandels und seiner Bekämpfung würde man sich eine differenziertere und „spitzere" Abrechnung des Leistungsaustauschs zwischen den Generationen wünschen. Hinzu kommt, dass die Eindämmung des Klimawandels weltweit ein geringeres Bevölkerungswachstum erfordern dürfte und damit überall zu Alterungsprozessen führen wird, die in allseits erträglicher Weise bewältigt werden müssen.
Was Generationengerechtigkeit ist, lässt sich aus ökonomischer Sicht nicht leicht präzisieren. Trotzdem ist man versucht zu fragen, mit welchem Recht die heute Älteren eigentlich von einer zahlenmäßig deutlich kleineren jungen Generation verlangen könnten, ihnen Renten zu finanzieren, die im Niveau konstant bleiben oder sogar wieder so hoch werden sollen wie vor zehn oder 20 Jahren - bei unverändertem Rentenalter womöglich auch noch für eine immer längere Laufzeit. Letztlich stellt sich dabei aber nicht nur ein mögliches Gerechtigkeitsproblem. Vielmehr ist abzusehen, dass eine solche Politik, selbst wenn sie in einer alternden Gesellschaft im Interesse der Mehrheit der Wähler:innen sein sollte (Sinn und Uebelmesser, 2002), auch aus anderen Gründen an Grenzen stößt.

\section{Maßstab II: internationale Wettbewerbsfähigkeit}

Den Einwand, dass stark steigende Beitragssätze die internationale Wettbewerbsfähigkeit in Deutschland produzierter Güter und die damit verbundenen Arbeitsplätze gefährden, wischt Niemeier $(2021,458)$ mit sehr punktuellen Hinweisen weg. So merkt er an, dass der Rentenbeitragssatz in Österreich mit 22,8\% derzeit über dem deutschen Wert von 18,6\% liegt, und unterschlägt, dass der Vergleich für den Gesamtsozialversicherungsbeitrag mit 36,45\% zu 39,95\% umgekehrt ausfällt. Internationale Vergleiche sind in diesem Kontext schwierig, weil die Struktur der Leistungen und der Mix aus Beitrags- und Steuerfinanzierung variieren. Bei der Summe lohnbezogener Steuern und Sozialabgaben liegt Deutschland mit einer Belastung von 49,0\% innerhalb der OECD aber schon jetzt - vor absehbaren Steigerungen - hinter Belgien auf dem zweiten Platz, und zwar mit großem Abstand zum OECD-Durchschnitt von 34,6\% (OECD, 2021). ${ }^{1} \mathrm{Zu}$ berücksichtigen ist zudem, dass Deutschland, vor allem wegen des schnellen und starken Geburtenrückgangs in den 1970er Jahren, in den nächsten 20 Jahren zu den am stärksten alternden Ländern der Erde gehören wird. Daher ist nicht damit zu rechnen, dass die Lohn(neben) kosten in anderen entwickelten Volkswirtschaften zeitlich parallel ganz ähnlich steigen wie hierzulande.

Die Alterung treibt neben den Beitragssätzen der GRV auch die der anderen Sozialversicherungen hoch. In ihrer Summe können diese bis $204050 \%$ überschreiten und bis 2080 auf $65 \%$ zugehen (Werding, 2018, 17, 30) - noch ohne Berücksichtigung der Lohnsteuer. Die Aufteilung dieser Beiträge auf Arbeitgeber:innen und Arbeitnehmer:innen ist aus ökonomischer Sicht zweitran-

1 Diese Berechnungen für 2020 beziehen sich auf Alleinstehende ohne Kinder mit durchschnittlichem Verdienst. Auch bei allen anderen, von der OECD betrachteten Fallkonstellationen liegt Deutschland mit an der Spitze. 
gig, wie Niemeier $(2021,458)$ nach anderslautenden Aussagen selbst bemerkt. In zukünftigen Lohnverhandlungen kann die Last daraus zwischen beiden Marktseiten hinund hergeschoben werden. Die Arbeitgeber:innen können sie prinzipiell auch weiterwälzen, z. B. auf ihre Kund:innen, sofern diese nicht die Anbieter:innen wechseln. Denkbar sind für die Zukunft daher Szenarien, in denen die Lohnkosten in Deutschland stark steigen, was den Absatz heimischer Güter im In- und Ausland erschwert, oder in denen die Nettolöhne stärker gedämpft werden als der Anstieg der Beitragssätze anzeigt. In diesem Fall sind es möglicherweise eher die deutschen Arbeitnehmer:innen, die im EU-Binnenmarkt abwandern oder sich Arbeit in Kopenhagen, Eindhoven, Luxemburg, Basel oder Prag suchen, mit zwei bis drei Tagen im „Home office“, über deren abgabenrechtliche Behandlung in der EU dann gestritten werden kann. In jedem Fall würde die Finanzierung des deutschen Sozialstaats in Mitleidenschaft gezogen.

Oberflächlich sind auch die Verweise von Niemeier (2021, 458) auf angesichts einer hohen Produktivität bisher eher niedrige Lohnstückkosten in Deutschland sowie auf geringe Beschäftigungseffekte von Lohnsteigerungen durch den 2015 eingeführten Mindestlohn. Gering waren in Deutschland in der Vergangenheit vor allem die Lohnstückkostensteigerungen, speziell vor der Wirtschafts- und Finanzkrise und im Kontext einer bis 2005 sehr ungünstigen Arbeitsmarktentwicklung. Seither haben sie sich etwa innerhalb der Eurozone eher normal bis überdurchschnittlich entwickelt (Herzog-Stein et al., 2020, 15-17). Das Niveau der Lohnstückkosten im exportorientierten Verarbeitenden Gewerbe ist nicht wirklich niedrig, auch wenn die derzeitigen Exportüberschüsse anzeigen, dass es hier unter Umständen noch gewisse Reserven gäbe, um Steigerungen zu verkraften. Dass Ökonom:innen Beschäftigungseinbußen durch den Mindestlohn vorab weit überschätzt hätten, wird von beteiligten Autoren (Knabe et al., 2020) anders eingeschätzt. Auch die von Niemeier ins Feld geführt Evaluation des IZA (2018) zeigt zwar keinen signifikanten Rückgang an Arbeitsplätzen, aber sehr wohl Reduktionen der Arbeitszeit, die in Vollzeitäquivalenten eine Größenordnung haben, die den Vorausschätzungen nahekommt.

\section{Schlussbemerkung}

Mit der Ansicht, dass höhere Beitragssätze der GRV kein Problem seien, solange die Nettolöhne trotzdem steigen, befindet sich Niemeier (2021) in guter Gesellschaft. 1995 legte Prognos im Auftrag des damaligen Bundessozialministers Blüm ein Gutachten vor (Eckerle und Schlesinger, 1995), in dem mit genau diesem Argument ein Anstieg des GRV-Beitragssatzes unter dem damaligen Recht auf $26,3 \%$ bis $28,7 \%$ bis 2040 als hinnehmbar eingeschätzt wird. Zwei Jahre später legte Blüm einen Gesetzentwurf vor, der mit der Einführung eines „demografischen Faktors" bei den Rentenanpassungen eine Abkehr vom tendenziell konstanten Netto-Rentenniveau einleitete, die von der nächsten, rot-grünen Bundesregierung mit den Reformen von 2001 und 2004 vollendet wurde.

Zusammen mit der 2007 beschlossenen Heraufsetzung der Regelaltersgrenze der GRV und vor dem Hintergrund einer stark verbesserten Arbeitsmarktentwicklung haben diese Reformen die Finanzierbarkeit der GRV bis etwa 2025 gesichert. Nun wird es Zeit für neue Reformen, die die akute Phase der demografischen Alterung in den Blick nehmen und - neben einer klugen Steuerung des Rentenniveaus - auch die weitere Entwicklung der Regelaltersgrenze und ergänzende, kapitalgedeckte Altersvorsorge unter besseren rechtlichen Rahmenbedingungen als bisher betreffen.

\section{Literatur}

Eckerle, K. und M. Schlesinger (1995), Perspektiven der Gesetzlichen Rentenversicherung für Gesamtdeutschland vor dem Hintergrund veränderter politischer und ökonomischer Rahmenbedingungen, Verband Deutscher Rentenversicherungsträger.

Fenge, R. und M. Werding (2004), Ageing and the tax implied in public pension schemes: Simulations for selected OECD countries, Fiscal Studies, 25, 159-200.

Herzog-Stein, A., P. Nüß, U. Stein und N. Albu (2020), Arbeits- und Lohnstückkostenentwicklung, IMK-Report, Nr. 158.

IZA (2018), Mindestlohn in Deutschland: Folgen für Beschäftigung, Arbeitszeit und Arbeitslosigkeit, IZA Research Report, Nr. 83.

Knabe, A., R. Schöb und M. Thum (2020), Prognosen und empirische Befunde: Wie groß ist die Kluft beim Mindestlohn wirklich?, Perspektiven der Wirtschaftspolitik, 21, 2529.

Kommission Verlässlicher Generationenvertrag (2020), Bericht der Kommission, Bundesministerium für Arbeit und Soziales (BMAS).

Niemeier, E. (2021), Beitragsfinanzierung im „demografiegestressten“ Rentensystem möglich, Wirtschaftsdienst, 6/2021, 454-460, https:// www.wirtschaftsdienst.eu/inhalt/jahr/2021/heft/6/beitrag/beitragsfinanzierung-im-demografiegestressten-rentensystem-moeglich.html (16. Juni 2021).

OECD (2021), Taxing Wages 2021, OECD.

Sachverständigenrat für die Begutachtung der gesamtwirtschaftlichen Entwicklung (2016), Zeit für Reformen (Jahresgutachten 2016/17), Statistisches Bundesamt.

Sinn, H.-W. und S. Uebelmesser (2002), Pensions and the path to gerontocracy in Germany, European Journal of Political Economy, 19, 153158.

Sozialbeirat (2004), Gutachten zum Rentenversicherungsbericht 2004, Bundestag-Drucksache. 15/4498.

Werding, M. (2018), Demographischer Wandel, soziale Sicherung und öffentliche Finanzen: Langfristige Auswirkungen und aktuelle Herausforderungen, Bertelsmann Stiftung.

Wissenschaftlicher Beirat beim BMF (2020), Der schwierige Weg zu nachhaltigen Rentenreformen (Gutachten).

Wissenschaftlicher Beirat beim BMWi (2021), Vorschläge für eine Reform der gesetzlichen Rentenversicherung (Gutachten). 


\section{Die beitragsfinanzierte Rente muss den Lebensunterhalt sichern}

Gegen die Forderung, den Lebensstandard sichernde Renten durch Beiträge zu ermöglichen, deren Belastungswirkung durch Einkommenssteigerungen überkompensiert wird, wendet Werding ein, dass das Rentenniveau nicht „,fixiert" werden dürfe; dass es in meinem JuniBeitrag nicht definiert worden sei; dass keine verlässlichen Maßstäbe für die Tragbarkeit der Beiträge formuliert worden seien; dass die intergenerationelle Lastverteilung fehlerhaft und die Wettbewerbsfähigkeit nicht belegt seien. Es seien vielmehr weitere Reformen notwendig, die die Reformen 2001 und 2004 weiterentwickeln. Werdings vorgetragene Argumente widerlegen die Notwendigkeit und Möglichkeit der Sicherung einer den Lebensstandard sichernden Rente durch tragbare Beiträge jedoch nicht.

\section{Verlässlicher Maßstab für tragbare Beitragssätze}

Die Überschrift des Kommentars von Werding erweckt den Eindruck, dass die Fixierung des Rentenniveaus das Problem sei. Es geht aber nicht um irgendeine Fixierung des Rentenniveaus, sondern um die Sicherung einer auskömmlichen Rente, die tendenziell den Lebensstandard sichert. Das Rentenniveau braucht in dem Beitrag im JuniHeft des Wirtschaftsdienst auch nicht definiert zu werden, weil er sich ausdrücklich auf Werdings Simulationsstudie und auf das dort verwendete 50-prozentige Standardrentenniveau bezieht (Werding, 2018). Wichtiger aber ist, dass das Rentenniveau nicht den Maßstab dafür bieten kann, ob sich seine Festlegung durchhalten lasse, wenn ein hoher Beitragssatz die Folge ist: ob der hohe Beitragssatz also tragbar sei. Der Maßstab dafür kann nur die Auswirkung des Beitragssatzes auf die Situation der Versicherten, also auf deren Tragbarkeit einerseits, und auf die Wettbewerbsfähigkeit der Unternehmen andererseits sein. Die Tragbarkeit hängt davon ab, ob die höheren Beiträge die Versicherten überfordern, d. h. ob sie der höheren Beiträge wegen ihre Lebenshaltung unzumutbar einschränken müssen. Diese Wirkung kann eindeutig an der Höhe des

(C) Der/die Autor:in(nen) 2021. Open Access: Dieser Artikel wird unter der Creative Commons Namensnennung 4.0 International Lizenz veröffentlicht (creativecommons.org/licenses/by/4.0/deed.de).

Open Access wird durch die ZBW - Leibniz-Informationszentrum Wirtschaft gefördert.

Dr. Ernst Niemeier war Lehrbeauftragter an der Hochschule Bremen.
Nettorealeinkommens abgelesen werden, das sich nach Abzug des gestiegenen Beitrags ergibt und das deshalb ein verlässlicher Maßstab für die Tragbarkeit ist.

An einem Beispiel wurde auf der Basis der von Werding unterstellten Annahmen über die Produktivitätsentwicklung und der von inm ermittelten Beitragssatzsteigerung aufgezeigt, dass eine Überforderung nicht eintreten kann. Denn das Nettorealeinkommen, der Wohlstand, steigt unter den Werdingschen Annahmen und unter Berücksichtigung seines Simulationsergebnisses deutlich an. Deshalb sind die Beiträge tragbar und ist das dazu gehörige Rentenniveau realistisch. Ich korrigiere allerdings die in dem Juni-Beitrag zugrunde gelegte Einkommensentwicklung bis 2070 auf 2080 und lege nun den von Werding für 2080 ermittelten Beitragssatz von 33,6\% zugrunde. Das Bruttorealeinkommen steigt dann - ausgehend vom angenommenen Einkommen von 2.500 Euro im Jahr 2000 auf 5.757,30 Euro. Der Rentenversicherungsbeitrag erhöht sich auf 967,23 Euro und das Nettorealeinkommen auf 4.790,07 Euro. Das entspricht einer Steigerung des Nettorealeinkommens um 111,25\%! Trotz des stark gestiegenen Beitragssatzes hat sich das Nettorealeinkommen mehr als verdoppelt. Die Beitragszahler können die höhere Beitragsbelastung deshalb problemlos tragen. Und selbst die Berücksichtigung einer Steigerung aller Sozialversicherungsbeiträge, die Werding in der Summe bis zu $65 \%$ für möglich hält, ändert an der Tragbarkeit nichts. Denn auch in diesem Fall würde das Nettorealeinkommen immer noch um 71,39\%, der Wohlstand also, deutlich steigen.

Weiter wendet Werding ein, dass es im Rentenrecht keinen Mechanismus gebe, der die Steigerung der Nettoentgelte sicherstelle, d. h. die Produktivitätssteigerungen in Einkommenssteigerungen umwandle. Das aber ist keine Frage des Rentenrechts. Die produktivitätsbezogenen Einkommenssteigerungen werden in Tarifverhandlungen durchgesetzt. Wie die Entwicklung nach 2010 es wieder zeigt, wird das im Trend auch erreicht. Während in diesem Beitrag also ein verlässlicher Maßstab für die Beitrags- und Rentenargumentation verwendet wird, lässt Werding für seine Behauptung, dass sich ein höheres Rentenniveau aus demografischen Gründen „kaum durchhalten“ lasse, einen Maßstab und eine Begründung vermissen (Werding, 2018).

\section{Begrenzte Eignung der dominierenden} ökonomischen Sicht

Die Überlegungen Werdings darüber, dass das (Standard-)Sicherungsniveau wenig über die tatsächliche Ver- 
sorgung der Rentner:innen aussage, müssen nicht weiter diskutiert werden, weil nicht das Rentenniveau der Maßstab für die Tragbarkeit steigender Beiträge ist. Außerdem sagt dieser statistische Wert sehr wohl etwas über die Tendenz der Versorgung der Rentner:innen aus. Deshalb spielt er in der politischen Diskussion eine Rolle und deshalb verwendet Werding inn in seinen Simulationen selbst.

Die von Werding weiterhin aufgeworfene Frage, warum viele Experten und Expertinnen und neuerdings auch die Mitglieder des Wissenschaftlichen Beirats des BMWi (2021) eine „Fixierung“ des Rentenniveaus für schwer realisierbar und keinesfalls für empfehlenswert halten, ist eine gute Frage. Allerdings gibt es auch Experten und Expertinnen, die den Mehrheitsmeinungen der Wissenschaftlichen Beiräte nicht zustimmen. So überschreibt der Ökonom Winfried Schmähl, langjähriger Vorsitzender des Sozialbeirats der Bundesregierung, in seinem 2018 erschienen Werk „Alterssicherungspolitik in Deutschland“ einen Unterabschnitt mit der Formulierung „Über die nur begrenzte Eignung des derzeit dominierenden ökonomischen Ansatzes für die Analyse der Alterssicherungspolitik" (Schmähl, 2018, 20). Schon 2012 stellte er fest, dass „der Abbau des Leistungsniveaus der GRV und der Ersatz durch kapitalmarktabhängige Alterssicherung nicht etwa die Lösung des Problems unzureichender Alterseinkommen, sondern dessen Ursache ist ... Der zentrale Ansatzpunkt für ein Umsteuern in der Alterssicherungspolitik ist folglich das Leistungsniveau in der GRV“ (Schmähl, 2012, 313).

\section{Generationengerechtigkeit}

Das bis 2080 deutlich steigende Nettorealeinkommen, das sich aus den Produktivitätsannahmen Werdings und aus seinen von ihm ermittelten Beitragssätzen ergibt, zeigt, dass diese Entwicklung nicht wirklich zu einem Problem der intergenerationellen Lastverteilung führt. Berechnungen „interner Renditen“ des gesetzlichen Rentensystems oder darin enthaltener „impliziter Steuern“ können den zu erwartenden Wohlstandsgewinn der jungen Generation nicht verschwinden lassen. Die Behauptung Werdings, dass für die junge Generation die Möglichkeiten substanziell eingeengt würden, eine ausreichende Altersvorsorge aufzubauen, ist nicht nachzuvollziehen. Denn die gestiegenen Beiträge, mit denen ein entsprechender eigentumsähnlicher Anspruch auf eine eigene Rente erworben wird, sichern auch der 2080 erwerbsaktiven Generation eine auskömmliche Altersversorgung. Der weitere Einwand Werdings, dass die Produktivitätsund Einkommenssteigerungen nicht sicher seien, ist zwar nicht zu bestreiten. Allerdings legt er seinen Simulationen genau die verwendeten Produktivitätssteigerungen zugrunde, woraus geschlossen werden muss, dass er sie für realistisch hält.
Das weitere Argument, dass die nicht voll voraussehbaren Folgen des Klimawandels zu beachten seien, ist zwar ebenfalls richtig. Eine Aufrechnung mit den Soziallasten und Sozialleistungen, eine "differenzierte“ und "spitze“ Abrechnung des Leistungsaustauschs zwischen den Generationen, ist aber nicht erlaubt. Denn es ist unumgänglich, die Rentner:innen zu alimentieren und menschenwürdig zu behandeln. Auch ein aus Klimaschutzgründen notwendig geringeres Bevölkerungswachstum und die sich daraus ergebende stärkere Alterung der Bevölkerung kann zu weiteren Belastungen führen. Sie kann aber nicht bedeuten, die Alten einfach abzuschreiben. Diese Diskussion der Generationengerechtigkeit vernachlässigt mit ihrer kritischen Sicht auf die Umlagefinanzierung, dass die junge Generation nicht nur die Renten der Rentner:innen zahlt, sondern mit ihrem Beitrag zugleich den Anspruch auf eine eigene auskömmliche Rente erwirbt.

Darüber hinaus verdeckt die Formulierung „mit welchem Recht die heute Älteren eigentlich von einer ... jungen Generation verlangen könnten, ihnen Renten zu finanzieren“, dass es eine gegenseitige Verantwortung der Generationen gibt und dass die Kindergeneration grundsätzlich sehr wohl auch daran interessiert ist, dass die Eltern im Alter menschenwürdig leben können. Übersehen wird ferner, dass die Elterngeneration nicht nur die Grundlagen für das Wohlstandsniveau der Kinder mitgelegt hat, sondern dass die Elterngeneration - sofern nur annähernd möglich - die Kindergeneration unterstützt. Schmähl weist darauf hin, „dass intrafamiläre Transfers überwiegend von Älteren zu Jüngeren erfolgen“, und zwar in Form von Geldleistungen, Realtransfers und durch Einsatz eigener Zeit sowie durch Schenkungen und Erbschaften (Schmähl, 2018, 1088 f.).

\section{Entlastungen für Beitragssatz möglich?}

Trotz der aufgezeigten grundsätzlichen Tragbarkeit höherer Beiträge zur Finanzierung auskömmlicher Renten kann und muss darüber diskutiert und muss geprüft werden, ob eine Entlastung, ob eine Verminderung der Beitragssätze zweckmäßig ist. Die Wissenschaftlichen Beiräte schlagen eine Verlängerung der Erwerbstätigkeitszeit vor, vielleicht angebunden an die eintretende Verlängerung der Lebenszeit und vielleicht auch differenziert nach stark belasteten Erwerbstätigkeiten. Diese Möglichkeit sollte im Hinblick auf eine vielleicht wünschenswerte Entlastung diskutiert und nicht von vornherein ausgeschlossen werden. Eine andere wirksame Entlastungsmöglichkeit wurde im Juni-Beitrag angesprochen. Sie würde nicht nur eine spürbare Beitragsentlastung bewirken können, sondern auch eine Ungerechtigkeit gegenüber den Arbeitnehmer:innen beseitigen. Es handelt sich um die „versicherungsfremden Leistungen“ (Meinhardt, 2018), die gesamtgesellschaftliche Aufgaben 
darstellen, deren Finanzierung sachlich falsch allein den Arbeitnehmer:innen aufgebürdet wird. Werding geht auf diese Entlastungsmöglichkeit bedauerlicherweise nicht ein.

\section{Wettbewerbsfähigkeit}

Die Wettbewerbsfähigkeit stellt den zweiten Maßstab für die Bewertung der Beiträge dar. Es muss gemessen werden, ob Rentenzahlungen, die eine bestimmte Beitragshöhe auslösen, den Wettbewerb nicht erschweren. Die österreichische Rentenpolitik mit ihren deutlich höheren Beiträgen und wesentlich höheren Renten kann in Verbindung mit der Betrachtung der Wettbewerbsfähigkeit der österreichischen Wirtschaft in Form ihrer Lohnstückkosten einen ersten Eindruck von den Möglichkeiten einer Erfolg versprechenden Rentenpolitik vermitteln. Werding wendet dagegen ein, dass die österreichischen Rentenversicherungsbeiträge zwar spürbar höher als die deutschen seien, dass aber die deutschen Gesamtsozialversicherungsbeiträge mit 39,95\% gegenüber denen Österreichs mit 36,45 \% höher lägen. Das ist zwar richtig, sagt über die Wettbewerbsfähigkeit der österreichischen Wirtschaft aber gar nichts aus. Die für den Wettbewerb relevanten Lohnstückkosten Österreichs lagen nach dem Jahr 2000 (= 100) immer erheblich über denen Deutschlands. Im Jahr 2018 wiesen sie für Deutschland den Wert von 122 auf, während Österreichs Wert bei 133 lag (Herzog-Stein et al., 2019, 13). Unter Berücksichtigung dieser Wettbewerbsposition ist das österreichische Rentensystem unter dem Gesichtspunkt der Wettbewerbsfähigkeit sehr wohl für Deutschland interessant.

Ein weiterer Einwand Werdings besagt, dass die Summe lohnbezogener Steuern und Sozialabgaben für Alleinstehende in Deutschland mit $49 \%$ relativ hoch sei und dass sie einen großen Abstand zum OECD-Durchschnitt von $34,6 \%$ aufweisen. Die OECD-Summen lohnbezogener Steuern und Daten, die nur für Alleinstehende so stark vom Mittelwert abweichen (OECD, 2021), stellen den sogenannten Steuerkeil dar, der die Belastung der Beschäftigten mit Steuern und Sozialabgaben ausdrückt. Dieser Steuerkeil beeinflusst aber die Wettbewerbsfähigkeit der Wirtschaft nicht und ist für diese Diskussion irrelevant. Wie schon ausgeführt, sind für die Wettbewerbsfähigkeit der Wirtschaft die Lohnstückkosten relevant. Sie drücken die durch Produktivitätssteigerungen bewirkte Relativierung der absoluten Kosten aus. So würde eine Steigerung der Lohnkosten in einer bestimmten Höhe für die Wettbewerbsfähigkeit irrelevant sein, wenn die Produktivität im gleichen Maße steigt. Die Betrachtung der gegenwärtigen Lohnstückkosten ist in dem hier diskutierten Zusammenhang trotz der Tatsache wichtig, dass sie den im Jahre 2080 relevanten Lohnstückkosten nicht entsprechen. Denn in der Vergangenheit und auch aktuell wird die Wett- bewerbsproblematik häufig unzulässig und interessengeleitet mit den absoluten Lohnnebenkosten als angeblich entscheidendem Wettbewerbsfaktor diskutiert, um sozialpolitisch motivierte Entscheidungen zu verhindern. Die Bedeutung von Produktivitäts- und Lohnstückkostenentwicklung für die Wettbewerbsfähigkeit aufzuzeigen, ist deshalb auch für 2080 wichtig, da deren Entwicklung Lohnnebenkostensteigerungen neutralisieren kann. Denn die Unternehmen lassen sich in den Tarifverhandlungen einen Teil der Produktivitätssteigerungen für ihre Kostensteigerungen zurechnen. Die Spekulationen Werdings über denkbare Szenarien stark steigender Lohnkosten in Deutschland, die er aus der Weiterwälzung von Lohnnebenkosten auf Kunden und Kundinnen ableitet, und die vermeintlich mögliche stärkere Dämpfung der Nettolöhne, die den Anstieg der Beitragssätze übertrifft, sind nur schwer nachzuvollziehen. Sie sind für die Frage der Wettbewerbsfähigkeit aber schon deshalb nicht relevant, weil die Produktivität vernachlässigt wird.

Der Vorwurf der Oberflächlichkeit meiner Ausführungen zur Entwicklung der deutschen Lohnstückkosten (Werding, 2021) beruht auf falschen oder fragwürdigen Behauptungen. Erstens sind die geringen oder gar negativen Lohnstückkostensteigerungen bis 2009 nicht durch eine hohe Produktivität zustande gekommen, sondern durch deutlich zu niedrige Lohnsteigerungen (Herzog-Stein et al., 2020, 13 und 15). Erst in den 2010er Jahren normalisierten sich die deutschen Lohnsteigerungen, ohne dass allerdings eine ausreichende „dynamische Lohnentwicklung" zustande gekommen wäre (Herzog-Stein et al., 2020). Zweitens war das deutsche Lohnstückkostenniveau im europäischen Vergleich nicht nur bis 2009 sehr niedrig, sondern blieb es trotz größerer Lohnsteigerungen auch in den Folgejahren. Auf der Basis des Jahres 2000 (=100) erreichten die deutschen Lohnstückkosten 2018 das Niveau von ca. 120. Die Niveaus Belgiens, Frankreichs, Italiens, der Niederlande, Österreichs und Spaniens lagen alle höher, und zwar von ca. 128 bis ca. 142 (Herzog-Stein et al., $2020,17)$. Drittens hebt Werding im Einklang mit dem Institut der deutschen Wirtschaft, das interessengeleitet ebenfalls mit den höheren Lohnstückkosten argumentiert, die relativ hohen Lohnstückkosten im exportorientierten Verarbeitenden Gewerbe hervor. Mit diesen Lohnstückkosten werden aber die Verflechtungen der Wirtschaftssektoren und die Vorleistungen des Dienstleistungsbereichs vernachlässigt. Deshalb stellen die Lohnstückkosten der Gesamtwirtschaft den richtigeren Indikator für die Wettbewerbsfähigkeit einer Volkswirtschaft dar. Dass die deutsche Wirtschaft trotz des von Werding als „nicht wirklich niedrig“ angesehenen Lohnstückkostenniveaus der Verarbeitenden Industrie Exportüberschüsse erzielt, veranlasst Werding zu der richtigen Bestätigung, es gäbe „unter Umständen noch gewisse Reserven ..., um Steigerungen [der 
Lohnstückkosten!] zu verkraften“ (Werding, 2021). Zu dem Beispiel der falsch vorausgeschätzten Beschäftigungseinbußen durch die Einführung des Mindestlohns weist Werding darauf hin, dass beteiligte Autoren das anders einschätzten. Die tatsächlich vom Mindestlohn verursachten Wirkungen herauszufinden, d.h. die Wirkungen anderer Einflussfaktoren auszuschließen, ist sehr schwierig. Wenn man die einschlägigen Untersuchungen studiert, kann man sich nur darüber wundern, wie waghalsig die Annahmen und Berechnungen sind. Und es ist auch nicht verwunderlich, dass unterschiedliche Untersuchungen unterschiedliche Ergebnisse erbringen. Da die Mindestlohndiskussion aber nur etwas über die Neigung aussagt, Lohnkosten als Wettbewerbsfaktor zu benutzen, er ohne Bezug auf die Produktivität jedoch kein Wettbewerbsfaktor ist, führe ich diese Diskussion hier nicht weiter.

In der Schlussbemerkung seines Kommentars bestätigt Werding, dass das Prognos-Institut 1995 die gleiche These vertreten habe, die in meinem Juni-Beitrag vertreten wurde; die These, dass höhere Beitragssätze tragbar seien, solange die Nettolöhne produktivitätsbedingt ansteigen. Die von Werding angeführte politische Entscheidung von 1997, einen „demographischen Faktor" einzuführen, mit dem die Absenkung des Rentenniveaus eingeleitet wurde, bedeutet jedoch nicht - wie Werding es offenbar sehen möchte -, dass die Prognos-Niemeier-These falsch ist. Der wirkliche Grund für den Schwenk in der Rentenpolitik ist ein ganz anderer. Ab Mitte der 1990er Jahre setzte eine Kampagne gegen die GRV in Form des Umlageverfahrens ein, weil das Umlageverfahren angeblich eine „tickende Zeitbombe" darstelle. Der damals in Mannheim lehrende Ökonom Axel Börsch-Supan sagte voraus, „dass das deutsche Alterssicherungssystem mit seiner starken Ausrichtung auf die Umlagefinanzierung am Rande des Kollapses stünde" (Schmähl, 2018, 1092). Durch den Dauerbeschuss der GRV beeinflusst, meinten Medien, öffentliche Meinung und Politiker:innen schließlich, eine Absenkung des gesetzlichen Rentenniveaus und einen Ersatz durch eine kapitalmarktgedeckte Alterssicherung realisieren zu müssen. Wie dieser problematische Paradigmenwechsel in der Rentenpolitik zu beurteilen ist, hat Schmähl - wie oben zitiert - beurteilt.

Die Überschrift über Werdings Kommentar weist schon darauf hin, dass er es für notwendig hält, das Rentenniveau weiter zu senken. Die angeblich gelungenen Reformen von 2001 und 2004, die das Rentenversicherungssystem aller- dings in ein Beitragssicherungssystem verwandelt haben, sollten seiner Meinung nach weiterentwickelt werden. Der teilweise Ersatz des gesetzlichen Rentenniveaus durch kapitalmarktgedeckte Renten verursacht aber schon deshalb für ein gegebenes Rentenniveau höhere Kosten für die Arbeitnehmer:innen, weil sie auch den Arbeitgeberanteil, der in der gesetzlichen Rentenversicherung zu leisten ist, tragen müssen. Als weiterer Nachteil kommt die fehlende Dynamisierung während der Rentenphase und das Kapitalmarktrisiko hinzu. Keine Gedanken werden darauf verwendet, ob das verminderte Rentenniveau die Rentner:innen am Leben erhalten kann. Werding hat der Beweisführung meiner Beiträge zwar widersprochen; der Tatsache, dass ein auskömmliches Rentenniveau mit tragbaren Beiträgen möglich ist. Er hat diese Argumentation aber nicht widerlegt. Ein auskömmliches Rentenniveau setzt allerdings voraus, dass zuvor ein ausreichend hohes Einkommen erzielt wurde. Sofern dies durch politische Fehler (unangebrachte Austeritätspolitik, Schaffung Niedriglohnsektor, etc.) oder systembedingte Probleme verhindert wurde, muss die Rente aus Steuermitteln aufgestockt werden (Niemeier, 2020).

\section{Literatur}

Herzog-Stein, A., Stein, U. und R. Zwiener(2019), Arbeits- und Lohnstückkostenentwicklung 2018 im europäischen Vergleich, IMK-Report, 149

Herzog-Stein, A., Stein, U. und R. Zwiener (2020), Arbeits- und Lohnkostenentwicklung, IMK-Report, 158.

Meinhardt, V. (2018), Versicherungsfremde Leistungen der Sozialversicherung - Expertise für das IMK, IMK-Study, 60, April.

Niemeier, E. (2021), Beitragsfinanzierung im „demografiegestressten“ Rentensystem möglich, Wirtschaftsdienst, 101(6), 454-460, https:// www.wirtschaftsdienst.eu/inhalt/jahr/2021/heft/6/beitrag/beitragsfinanzierung-im-demografiegestressten-rentensystem-moeglich.html (6. Juli 2021).

Niemeier, E. (2020), Politische Ursachen für Rentenprobleme und Altersarmut erfordern grundlegende Reformen, Wirtschaftsdienst, 100(8), 597-599, https://www.wirtschaftsdienst.eu/inhalt/jahr/2020/heft/8/ beitrag/politische-ursachen-fuer-rentenprobleme-und-altersarmuterfordern-grundlegende-reformen.html (6. Juli 2021).

OECD (2021), Taxing Wages, OECD Paris.

Schmähl, W. (2012), Von der Rente als Zuschuss zum Lebensunterhalt zur „Zuschuss-Rente“, Wirtschaftsdienst, 92(5), 304-313, https://www. wirtschaftsdienst.eu/inhalt/jahr/2012/heft/5/beitrag/von-der-renteals-zuschuss-zum-lebensunterhalt-zur-zuschuss-rente-weichenstellungen-in-120-jahren-ge.html (6. Juli 2021).

Schmähl, W. (2018), Alterssicherungspolitik in Deutschland.

Werding, M. (2018), Demographischer Wandel, soziale Sicherung und öffentliche Finanzen: Langfristige Auswirkungen und aktuelle Herausforderungen.

Werding, M. (2021), Warum das Rentenniveau nicht fixiert werden sollte: ein Kommentar, Wirtschaftsdienst, 101(7).

Wissenschaftlicher Beirat beim BMWi, (2021), Vorschläge für eine Reform der gesetzlichen Rentenversicherung (Gutachten), BMWi Berlin.

Title: Debate about Pensions - Reply and Response

Abstract: In its June 2020 issue, Wirtschaftsdienst published an article entitled „Beitragsfinanzierung im , demografiegestressten“ Rentensystem möglich" by Ernst Niemeier. Martin Werding takes a different view in a reply, and Ernst Niemeier explains his point of view in a response.

JEL Classification: H53, H55, I38, J14 